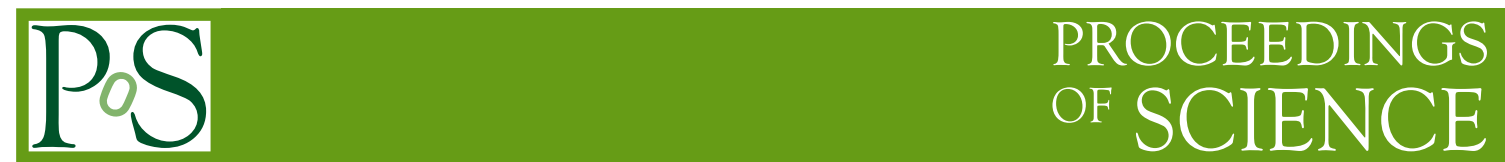

\title{
Neutrino factory without charge ID
}

\section{PATRICK HUBER*}

\section{CERN}

E-mail: pahuberevt.edu

In this contribution we show that a high precision separation of $v / \bar{v}$ events in a low energy neutrino factory is not necessarily required to achieve the physics goals of such a facility.

10th International Workshop on Neutrino Factories, Super beams and Beta beams June 30 - July 52008

Valencia, Spain

${ }^{*}$ Speaker. 
A neutrino factory exploits the so called wrong sign muon signal to look for oscillations of $\bar{v}_{e} \rightarrow \bar{v}_{\mu}^{1}$ [1]. This oscillation channel is well know to be sensitive to $\sin ^{2} 2 \theta_{13}$, CP violation and the mass hierarchy. The experimental challenge in a neutrino factory experiment, besides from making the neutrino beam, is to disentangle the rare wrong sign muons from the abundant right sign muons. Usually, a magnetic field is proposed to charge separate the muon and anti-muon with very high accuracy; for a review of this option, see [2]. Clearly, magnetic charge separation works best for muons with a large momentum well exceeding $1 \mathrm{GeV}$. One reason is, that a high momentum muon has a long, well defined track which provides many measurements along the trajectory, thus making it easy to determine the curvature. Another reason is, that the likelihood that the most energetic particle produced in a deep inelastic scattering event is not a muon decreases with increasing momentum of that most energetic particle. In summary, this has lead to an optimized neutrino factory setup with a muon energy of $25 \mathrm{GeV}$, which in turn necessitates baselines of several thousand kilometers [3]. However, it has been recognized that the performance of a neutrino factory at large $\sin ^{2} 2 \theta_{13} \geq 10^{-2}$ is not vastly superior in comparison to alternative technologies like superbeams or beta beams [3,4]. This fact has stipulated efforts to develop an entry level neutrino factory with a lower muon energy, the so called low energy neutrino factory $[5,6]$ with muon energies around $5 \mathrm{GeV}$. At those energies, charge separation in a magnetic field becomes very challenging, therefore advanced detector concepts like magnetized fully active scintillating detector have been proposed. The high channel count and large magnetized volume makes it appear unlikely that detectors of this type much larger than $10-20 \mathrm{kt}$ are feasible. In this note, we would like to explore an alternative detection scheme at a low energy neutrino factory which does not involve magnetic fields. The details of these idea can be found in [7]. This technique is based on two, simple observations: At the first oscillation maximum most of the right sign muons have oscillated into $v_{\tau}$, while the wrong sign muon signal peaks. Thus oscillation itself provides a sizable enhancement of the signal to background ratio. The other observation is, that neutrinos and anti-neutrinos ${ }^{2}$ behave differently in charged current interactions with matter and produce different final states. Thus, there are potentially some detectable differences between right and wrong sign muon events even in the absence of a magnetic field.

The $v_{\mu}$ survival probability $P_{\mu \mu}$ vanishes approximately for $\frac{\Delta m_{31}^{2} L}{4 E}=\pi / 2$. At the same energy, the leading term in the appearance probability $P_{e \mu}$ will be maximal, hence the ratio of the wrong sign signal from $P_{e \mu}$ to the right sign background from $P_{\mu \mu}$ becomes very large. In order to exploit this effect a very good energy resolution is crucial otherwise it will be washed out by events from outside the valley in $P_{\mu \mu}$ which migrate into the valley due to the energy resolution. Fortunately, for neutrino energies as low as a few $\mathrm{GeV}$, there is a sizable contribution of quasi-elastic scattering to the total charged current cross section. Using these events good energy resolution can be obtained without accurate hadron calorimetry. In the following, we will only consider quasi-elastic events. Also, the neutrino energy should be such that the amount of $\tau$ leptons produced from $v_{\mu} \rightarrow v_{\tau}$ oscillation stays small enough, such that muonic $\tau$ decays to do not fill the dip in $P_{\mu \mu}$. We conclude, that muon energies in the range $4-5 \mathrm{GeV}$ are optimal.

\footnotetext{
${ }^{1}$ We only write the result for $\mu^{-}$stored, but imply that there is the CP conjugate process stemming from $\mu^{+}$decays.

${ }^{2}$ more precisely, left and right handed neutrinos
} 
Quasi-elastic neutrino reactions can schematically described by

$$
v_{x}+N \rightarrow l_{x}^{-}+p+N^{\prime} \quad \text { and } \quad \bar{v}_{x}+N \rightarrow l_{x}^{+}+n+N^{\prime},
$$

where $l_{x}$ denotes a charged lepton with $x$ being $\mu$ or $e$ and $N$ is the nucleus. The are a number of differences between the reactions of neutrino and anti-neutrinos beyond the charge of the outgoing lepton. For muon neutrino events, the lifetime of the resulting muon is different for $\mu^{+}$and $\mu^{-}$. The $\mu^{+}$lifetime corresponds to the one in vacuum, but the $\mu^{-}$can be bound to a nucleus by electromagnetic interaction and subsequently undergo muon capture onto the nucleus, thus shortening its lifetime. The capture probability in common detector materials [8] is: 8\% for Carbon, i.e. liquid scintillator $^{3}, 18 \%$ for Oxygen, i.e. water ${ }^{3}$ and $76 \%$ for Argon. This effect has been used by the Kamiokande collaboration to determine the charge ratio of cosmic ray muons with an accuracy of $6 \%$ [9]. Another difference is, the angular distribution of the outgoing lepton with respect to the incoming neutrino. For anti-neutrinos this distribution is more forward peaked than for neutrinos. This angular difference is largest for incoming energies around $1 \mathrm{GeV}$. The most obvious difference, of course, is the nature of the outgoing nucleon: a proton for neutrino events and a neutron for anti-neutrino events. Both nucleons can, at least in principle, be tagged and distinguished. Proton tagging can be quite reliably achieved in liquid Argon [10]. Proton tagging in water is difficult, unless the proton is quite energetic, thus neutron tagging may be more efficient. Neutron tagging in water can may become possible by adding about $0.2 \%$ Gadolinium [11]. The neutron capture cross section of Gadolinium is so large that almost all neutrons will be captured on Gadolinium. In this process a $\gamma$ cascade with about $8 \mathrm{MeV}$ is emitted, which can be clearly identified.

These consideration indicate the potential for separation of neutrino and anti-neutrino events even without the use of a magnetic field. The quantitative question, how pure the resulting event samples could be, requires careful and detailed study for each of the discussed effects in combination with a particular type and design of detector. This, however, is beyond the scope of the present work. Instead, we chose to parametrize the efficiency of the $v / \bar{v}$-separation in a simple way, using one parameter $p$ and show our results as a function of this parameter. We define two event samples: an anti-neutrino-like sample $N_{1}$ and a neutrino-like sample $N_{2}$

$$
\begin{aligned}
& N_{1}^{i}=\frac{1-p}{2} N_{v}^{i}+\frac{1+p}{2} N_{\bar{v}}^{i} \\
& N_{2}^{i}=\frac{1+p}{2} N_{v}^{i}+\frac{1-p}{2} N_{\bar{v}}^{i},
\end{aligned}
$$

where a value of $p=0$ corresponds to no separation at all, whereas $p=1$ corresponds to perfect separation. For $p=1, N_{1}$ would be a pure anti-neutrino sample and $N_{2}$ would be a pure neutrino sample. The chosen parametrization is an over-simplification and considerable deviations from it are to be expected in a real experiment. However, for the purpose of establishing the approximate level of required $v / \bar{v}$ separation, this will suffice.

Table 1 contains a list of detectors and their performance which will be used to compare the obtainable sensitivities of various possible setups as a function of $p$ and the detector size. The TASD (totally active scintillator detector) corresponds to the detector initially proposed for a low energy neutrino factory and is magnetized to about $0.5 \mathrm{~T}$ [6]. It will serve as our benchmark, only setups having a similar or better performance can be considered as reasonable alternatives. For the

\footnotetext{
${ }^{3}$ The muon capture rate on Hydrogen is negligibly small.
} 


\begin{tabular}{l|ccc}
\hline \hline & TASD [6] & WC [12] & LAr [13] \\
\hline fiducial mass [kt] & 20 & 500 & 100 \\
efficiency & 0.73 & $0.9^{4}$ & 0.8 \\
magnetized & yes & no & no \\
$\Delta E$ at $2.5 \mathrm{GeV}[\mathrm{MeV}]$ & 165 & $300^{5}$ & 165 \\
$p$ for muons & 0.999 & $0-0.7$ & $0.7-0.9$ \\
$p$ for electrons & 0 & 0 & $0.7-0.9$ \\
\hline \hline
\end{tabular}

Table 1: Summary of relevant detector parameters. Further details of our simulations can be found the references given in the first line of the table.
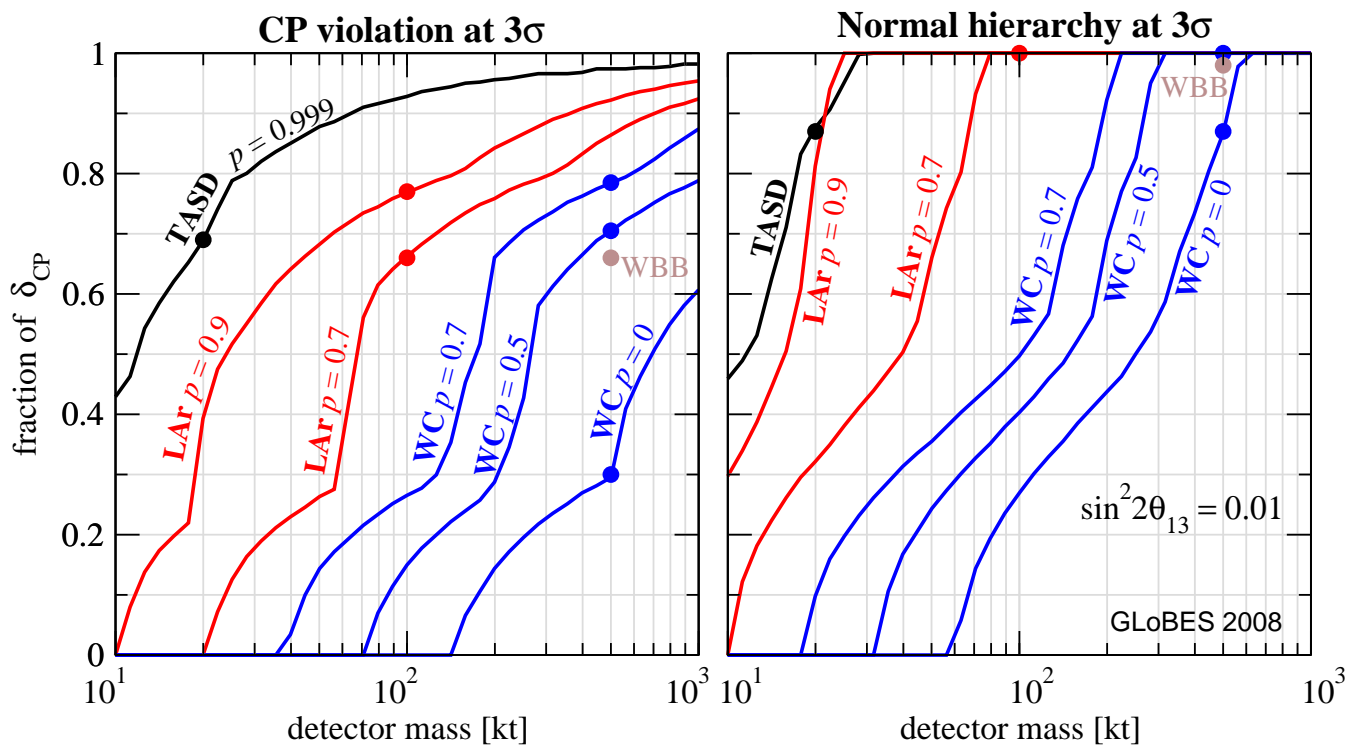

Figure 1: Fraction of $\delta$ as function of the detector mass for which CP violation (left hand panel) or the mass hierarchy can be discovered (right hand panel) at $3 \sigma$. confidence level for different experiments as described in table 1 for $\sin ^{2} 2 \theta_{13}=0.01$. The numbers next to the lines correspond to different values of the $v / \bar{v}$ separation coefficient $p$ as defined in equation 2 Figure taken from [7].

neutrino factory we use a stored muon energy $E_{\mu}$ of $5 \mathrm{GeV}$ and total of $10^{22}$ useful muon decays, equally divided into $\mu^{-}$and $\mu^{+}$running. This luminosity corresponds to 10 years total running time of the baseline setup of the International Design Study for a neutrino factory [14]. We assume a baseline of $1290 \mathrm{~km}$, which corresponds to the distance from Fermilab to the Deep Underground Science and Engineering Laboratory (DUSEL) at Homestake.

To calculate the sensitivities we will use $\Delta m_{31}^{2}=2.5 \cdot 10^{-3} \mathrm{eV}^{2}, \sin ^{2} \theta_{23}=0.5, \Delta m_{21}^{2}=7.6$. $10^{-5} \mathrm{eV}^{2}$ and $\sin ^{2} \theta_{12}=0.3$. For $\theta_{13}$ and $\delta$ we assume that they have to be determined by the experimental setups considered. The analysis is performed with GLoBES [15, 16] using a 4\% error on the solar parameters $\Delta m_{21}^{2}$ and $\sin ^{2} \theta_{12}$ and a $5 \%$ error on the matter density. We assume a $2.5 \%$ systematic error on each signal. All sensitivities are evaluated at the $3 \sigma$ confidence level for 1 degree of freedom, i.e. $\Delta \chi^{2}=9$.

The left hand panel of figure 1 shows that, depending on the detector type and level of $v / \bar{v}$ - 
separation, a larger detector mass is needed to achieve the same sensitivity as the usual magnetized TASD with a fiducial mass of $20 \mathrm{kt}$. For the WC, we find equivalent masses in the range from $200-500 \mathrm{kt}$ for $p=0.7-0.5$ and for the LAr the mass range is from $50-110 \mathrm{kt}$ for $p=0.9-0.7$. The equivalent masses increase for smaller values of $\theta_{13}$ and for $\sin ^{2} 2 \theta_{13}=0.003$, the equivalent mass ranges become $m=500-900 \mathrm{kt}$ for WC and $m=110-300 \mathrm{kt}$ for LAr.

In conclusion, we have shown that non-magnetized detectors can compensate their lack of high precision $v / \bar{v}$-separation by their larger mass for not too small values of $\sin ^{2} 2 \theta_{13} \geq 0.003$. Non-magnetized, large detectors have a rich physics program on their own and thus may already be existing when a neutrino factory is considered. Thus, the here proposed scheme of a low energy neutrino factory with a non-magnetized detector may serve as the inital step towards a high-energy neutrino factory.

Acknowledgments This work was supported by the European Community-Research Infrastructure Activity under the FP6 "Structuring the European Research Area" program (CARE, contract number RII3-CT-2003-506395).

\section{References}

[1] S. Geer, Phys. Rev. D 57 (1998) 6989 [Erratum-ibid. D 59 (1999) 039903] [arXiv:hep-ph/9712290].

[2] T. Abe et al. [ISS Detector Working Group], arXiv:0712.4129 [physics.ins-det].

[3] J. S. Berg et al. [ISS Accelerator Working Group], arXiv:0802.4023 [physics.acc-ph].

A. Bandyopadhyay et al. [ISS Physics Working Group], arXiv:0710.4947 [hep-ph].

[4] P. Huber, M. Lindner, M. Rolinec and W. Winter, Phys. Rev. D 74 (2006) 073003 [arXiv:hep-ph/0606119].

[5] A. D. Bross, M. Ellis, S. Geer, O. Mena and S. Pascoli, Phys. Rev. D 77 (2008) 093012 [arXiv:0709.3889 [hep-ph]].

[6] S. Geer, O. Mena and S. Pascoli, Phys. Rev. D 75 (2007) 093001 [arXiv:hep-ph/0701258].

[7] P. Huber and T. Schwetz, , Phys. Lett. B. in press, arXiv:0805.2019 [hep-ph].

[8] T. Suzuki, D. F. Measday and J. P. Roalsvig, Phys. Rev. C 35 (1987) 2212.

[9] M. Yamada et al., Phys. Rev. D 44 (1991) 617.

[10] F. Arneodo et al. [ICARUS-Milano Collaboration], Phys. Rev. D 74 (2006) 112001 [arXiv:physics/0609205].

[11] J. F. Beacom and M. R. Vagins, Phys. Rev. Lett. 93 (2004) 171101 [arXiv:hep-ph/0309300].

[12] V. Barger, M. Dierckxsens, M. Diwan, P. Huber, C. Lewis, D. Marfatia and B. Viren, Phys. Rev. D 74 (2006) 073004 [arXiv:hep-ph/0607177].

[13]

[13] V. Barger et al., arXiv:0705.4396 [hep-ph].

[14] S. Berg, et al., Tech. Rep. IDS-NF-002, IDS-NF, 2008.

[15] P. Huber, M. Lindner and W. Winter, Comput. Phys. Commun. 167, 195 (2005) [arXiv:hep-ph/0407333].

[16] P. Huber, J. Kopp, M. Lindner, M. Rolinec and W. Winter, Comput. Phys. Commun. 177, 432 (2007) [arXiv:hep-ph/0701187]. 